\title{
Continuous positive air pressure treatment reduces serum advanced glycation end products in patients with obstructive sleep apnoea syndrome: a pilot study
}

\author{
*Kazuhiko Kotani ${ }^{a, b, c}$ Satoshi Kimurad, Ichiro Komadae, Naoki Sakane ${ }^{a}$, Alejandro Gugliucci ${ }^{\mathrm{b}}$ \\ a Division of Preventive Medicine, Clinical Research Institute for Endocrine and Metabolic Disease, National Hospital Organization \\ Kyoto Medical Center, Kyoto, Japan \\ b Glycation, Oxidation and Disease Laboratory, Touro University-California, Vallejo, CA, USA \\ Department of Clinical Laboratory Medicine, Jichi Medical University, Shimotsuke-City, Tochigi, Japan \\ d Department of Clinical Laboratory, Showa University Northern Yokohama Hospital, Yokohama-City, Japan \\ e Department of Otorhinolaryngology, Shiga Hospital of Social Insurance, Shiga, Japan
}

Received 27th April 2010; revised 18th November 2010; accepted 28th December 2010; online22nd March 2011

\begin{abstract}
Nasal continuous positive air pressure (nCPAP) treatment may favourably affect serum levels of advanced glycation end products (AGEs) in patients with obstructive sleep apnoea syndrome (OSAS). At baseline, OSAS patients had significantly higher levels of AGEs than controls. Six months after nCPAP initiation, AGEs decreased significantly. nCPAP treatment could lower AGEs in patients with OSAS.

(C) 2011 Primary Care Respiratory Society UK. All rights reserved.

K Kotani et al. Prim Care Respir J 2011; 20(3): 336-337

http://dx.doi.org/10.4104/pcrj.2011.00021
\end{abstract}

Keywords oxidative stress, advanced glycation, AGE, sleep apnoea, nasal CPAP

\section{Introduction}

Advanced glycation end products (AGEs) are implicated in the pathogenesis of diabetic complications as well as kidney failure, with an important role in atherogenesis. ${ }^{1-3}$ Enhanced production of reactive oxygen species (ROS) by the mitochondrial electron transport chain leads to formation of intracellular AGEs that can be blocked by normalising mitochondrial superoxide production. ${ }^{3}$ AGEs are formed not only in conditions of hyperglycaemia but also in states of enhanced oxidative stress. ${ }^{3}$ A recent report has shown that the serum AGE concentration is increased in subjects with obstructive sleep apnoea syndrome (OSAS), which is considered a condition with oxidative stress. ${ }^{4}$ In OSAS, frequent episodes of upper airway obstruction occur during sleep and lead to significant hypoxia. ${ }^{5}$ The ensuing recurring changes in arterial oxygen saturation and hypoxia/reoxygenation tilts the oxidative balance towards an excess of oxygen-free radicals. Biomarkers of oxidative stress may thus be consistently elevated in this type of patient.

OSAS is a common disease and is strongly associated with the risk of cardiovascular disease (CVD); recognition of the pathophysiology of OSAS is therefore crucial in the primary care setting. ${ }^{5,6}$ Treatment with nasal continuous positive airway pressure (nCPAP) improves sleep and daytime sleepiness by reducing the hypoxic cycles (reducing ROS production), ${ }^{7}$ but its effects on CVD risk - while promising - remain incompletely established. ${ }^{5.8}$ We hypothesised that nCPAP has a beneficial effect on the oxidative phase of advanced glycation and therefore on serum AGE levels as biomarkers. In this preliminary study we measured AGE levels in a cohort of patients with OSAS at baseline and 6 months after initiation of nCPAP treatment compared with age- and sex-matched control subjects.

\section{Methods}

Ten Japanese patients with OSAS ( 5 men $/ 5$ women; mean \pm SE age $52 \pm 4$ years) and 30 age- and sex-matched controls (mean $52 \pm 4$ years) were studied. We included the patients with characteristic symptoms such as daytime somnolence, chronic fatigue and restless sleep as well as an apnoea plus hypopnoea score (apnoeahypopnoea index) of $\geq 20 / \mathrm{hr}$ of sleep on polysomnography. All patients were non-diabetics and they did not change their exercise

* Corresponding author: Dr Kazuhiko Kotani, Department of Clinical Laboratory Medicine, Jichi Medical University, 3311-1 Yakushiji, Shimotsuke-City, Tochigi 329-0498, Japan. Tel: +81-285-58-7386 Fax: +81-285-44-9947 E-mail: kazukotani@jichi.ac.jp 
habits or medication during the study. Subjects with a history of cardiovascular, cerebrovascular, renal, thyroid, psychiatric or infectious diseases were excluded. In control subjects, OSAS was clinically excluded by Kapuniai's criteria. ${ }^{9}$ The study was approved by the institutional ethics committees and each subject gave informed consent.

At baseline, after an overnight fast, body weight, serum AGEs and lipid panels were measured. For AGEs, fluorescence intensity was recorded at the emission maximum (440nm) upon excitation at $350 \mathrm{~nm}$ and at $335 / 385$ for pentosidine fluorescence. ${ }^{1,2,10}$ Fluorescence intensity is expressed in arbitrary units (AU). We used a SPECTRAmax Gemini XPS spectrofluorometer with SOFTmax PRO software (Molecular Devices, Sunnyvale, CA, USA). The measurements in the OSAS patients were repeated after 6 months of nCPAP treatment.

Differences between groups were compared by paired or unpaired $t$ test. The correlations between bivariables were examined by Spearman's rank correlation. A $p$ value of $<0.05$ was considered significant.

\section{Results}

The mean baseline characteristics of the patients with OSAS were: weight $72.7 \pm 2.9 \mathrm{~kg}$, total cholesterol $5.74 \pm 0.25 \mathrm{mmol} / \mathrm{L}$, triglycerides $1.74 \pm 0.22 \mathrm{mmol} / \mathrm{L}$, high-density lipoprotein cholesterol $1.40 \pm 0.14 \mathrm{mmol} / \mathrm{L}$. At baseline, AGE levels in patients with OSAS were higher than in controls $(82.8 \pm 26.0$ vS . $64.2 \pm 12.2 \mathrm{AU}, p=0.001)$ and pentosidine fluorescence was similar to controls $(37.4 \pm 10.5$ vs. $36.1 \pm 9.2 \mathrm{AU}, \mathrm{p}>0.05)$. AGES were $29 \%$ higher in OSAS patients than in control subjects.

After nCPAP treatment the patients consistently and significantly showed decreased levels of AGEs (68.9 \pm 4.2 vs. $82.8 \pm 26.0 \mathrm{AU}$ at baseline, $\mathrm{p}=0.03$ ). Similar changes were found for pentosidine fluorescence $(28.3 \pm 10.2$ vs. $37.4 \pm 10.5 \mathrm{AU}$ at baseline, $p=0.01)$ - that is, after nCPAP treatment, AGEs decreased by $17 \%$ (range 1-42\%) and pentosidine fluorescence decreased by $24 \%$ (range $1-40 \%$ ). No significant correlations were found with other parameters measured.

\section{Discussion}

In this study, AGE levels were significantly higher in patients with OSAS than in controls; this is in agreement with the only previous paper published on this subject. ${ }^{5}$ More importantly, our data strongly suggest that nCPAP treatment could lower serum AGE levels in OSAS patients. To our knowledge, this is the first report showing this beneficial effect of nCPAP. This finding may be partly explained by the fact that $\mathrm{nCPAP}$ (which could minimise the cycles of hypoxia/reoxygenation) has favourable effects on the production of ROS and oxidative stress. . The interaction of AGEs with their main receptors is largely a pro-inflammatory process; accordingly, therapies that lower the AGE burden may prove to be beneficial in the prevention of OSAS-related complications. AGEs are candidate factors in the enhanced risk of CVD development in OSAS patients, so our results may partly support this mechanism. A limitation of this study is the small number of patients studied; nevertheless, it serves as proof-ofprinciple evidence for an effect of nCPAP treatment in lowering serum levels of AGEs. More research on the effects of nCPAP and the role of AGEs in a larger cohort of OSAS patients using more sensitive and specific AGE biomarkers is warranted.

\section{Conflicts of interest}

There are no conflicts of interest in relation to the research presented.

\section{Funding}

This study was funded in part by Touro University and was partly supported by Grant-in-Aid for Scientific Research from the Ministry of Education, Culture, Sports, Science and Technology (KK and SK) in Japan.

\section{References}

1. Monnier VM, Kohn RR, Cerami A. Accelerated age-related browning of human collagen in diabetes mellitus. Proc Natl Acad Sci USA 1984;81:583-7.

2. Dyer DG, Blackledge JA, Thorpe SR, Baynes JW. Formation of pentosidine during nonenzymatic browning of proteins by glucose. Identification of glucose and other carbohydrates as possible precursors of pentosidine in vivo. J Biol Chem 1991;266:11654-60.

3. Ramasamy R, Yan SF, Herold K, Clynes R, Schmidt AM. Receptor for advanced glycation end products: fundamental roles in the inflammatory response: winding the way to the pathogenesis of endothelial dysfunction and atherosclerosis. Ann NY Acad Sci 2008;1126:7-13. http://dx.doi.org/10.1196/annals.1433.056

4. Tan KC, Chow WS, Lam JC, et al. Advanced glycation endproducts in nondiabetic patients with obstructive sleep apnea. Sleep 2006;29:329-33.

5. Shahar E, Whitney CW, Redline S, et al. Sleep-disordered breathing and cardiovascular disease: cross-sectional results of the Sleep Heart Health Study. Am J Respir Crit Care Med 2001;163:19-25.

6. Lieberman JA 3rd. Obstructive sleep apnea (OSA) and excessive sleepiness associated with OSA: recognition in the primary care setting. Postgrad Med 2009;121:33-41. http://dx.doi.org/10.3810/pgm.2009.07.2027

7. Schulz R, Mahmoudi S, Hattar K, et al. Enhanced release of superoxide from polymorphonuclear neutrophils in obstructive sleep apnea. Impact of continuous positive airway pressure therapy. Am J Respir Crit Care Med 2000;162(2 Pt 1):566-70.

8. Lavie L, Vishnevsky A, Lavie P. Evidence for lipid peroxidation in obstructive sleep apnea. Sleep 2004;27:123-8.

9. Kapuniai LE, Andrew DJ, Crowell DH, Pearce JW. Identifying sleep apnea from self-reports. Sleep 1988;11:430-6.

10. Gugliucci A, Mehlhaff K, Kinugasa E, et al. Paraoxonase-1 concentrations in end-stage renal disease patients increase after hemodialysis: correlation with low molecular AGE adduct clearance. Clin Chim Acta 2007;377:213-20. http://dx.doi.org/10.1016/j.cca.2006.09.028

\section{Available online at http://www.thepcrj.org}

